\title{
Responsabilidad social y la gestión de calidad: Empresa Peruana de Seguros
}

\section{Social responsibility and quality management: Peruvian insurance company}

Dr. Jaime Agustín Sánchez-Ortega es jefe de la unidad de investigación del Instituto de Gobierno y Gestión Pública, Universidad de San Martín de Porres, Lima-Perú (asesorjaime.s@gmail.com) (https://orcid.org/0000-0002-2916-7213)

Lic. Alejandra Seminario-Polo es analista de planeamiento de la empresa Pacífico Seguros, Perú (alejandra.seminario19@gmail.com) (https://orcid.org/0000-0002-5308-4901)

Mg. Abel Marcial Oruna-Rodríguez es docente en la Universidad Privada del Norte (UPN) Los Olivos, Facultad de Negocios, Lima, Perú (abel.oruna@upn.pe) (https://orcid.org/0000-0001-6380-1014)

\begin{abstract}
Resumen
Para ser sostenible en el tiempo, la responsabilidad social empresarial debe incidir en su entorno y sus colaboradores en tiempos de incertidumbre. Una crisis en los sistemas de salud, políticos y económicos en Perú se ha desencadenado como resultado de la COVID-19. El propósito de esta investigación fue determinar la relación entre la responsabilidad social y la gestión de calidad en una empresa peruana de seguros. La metodología utilizada para lograr el objetivo fue de enfoque cuantitativo, diseño no experimental y de alcance transversal. Se encuestaron a 331 colaboradores en la ciudad de Lima. Se aplicó como instrumento un cuestionario validado por juicio de expertos y con una confiabilidad de Cronbach $\alpha>$ 0,96. Los resultados evidencian que la empresa apoya a las comunidades menos privilegiadas en campañas de salud y nutrición a través de colaboradores voluntarios de los programas de responsabilidad social empresarial que contribuyen al cuidado del medio ambiente. En conclusión, existe una relación significativa entre las variables estudiadas; sin embargo, la alta dirección de la empresa aseguradora es consciente de que aún falta trabajar en la gestión del conocimiento y estrategias para conseguir la participación de sus colaboradores y proveedores en las labores de responsabilidad social en tiempos de pandemia, que permitan mayores niveles de competencia.
\end{abstract}

\begin{abstract}
To be sustainable over time, corporate social responsibility must influence its environment and its collaborators in times of uncertainty. A crisis in the health, political, and economic systems in Peru has been triggered as a result of COVID-19. The purpose of this study is to determine the relationship between social responsibility and quality management in a Peruvian insurance company. The methodology used to achieve the objective was a quantitative approach, cross-sectional and non-experimental design. A sample of 331 employees in the city of Lima was surveyed. The survey was applied as an instrument through the validation of contents by expert judgment and reliability of Cronbach Alpha>0.96. The results show that the company supports the less privileged communities in health and nutrition campaigns through voluntary collaborators of corporate social responsibility programs who contribute to caring for the environment. We conclude that there is a significant relationship between the variables studied, however, the insurance company is aware that it is still necessary to work on knowledge management and strategies to get the participation of its collaborators and suppliers in social responsibility tasks in times of pandemic, which allow higher levels of competition.
\end{abstract}

\section{Palabras clave I keywords}

Responsabilidad social, gestión de la calidad, comunidad, medio ambiente, gestión del conocimiento, desempeño laboral, COVID-19.

Social responsibility, Quality management, community, environment, knowledge management, job performance, COVID-19.

Cómo citar: Sánchez-Ortega, J.A., Seminario-Polo, A., y Oruna-Rodríguez, A.M. (2021). Responsabilidad social y la gestión de calidad: empresa peruana de Seguros. Retos Revista de Ciencias de la Administración y Economía, 11(21), pp. 117-130. https://doi.org/10.17163/ret.n21.2021.07. 


\section{Introducción}

La pandemia de la COVID-19 ha complicado la forma de vida que desarrollaban personas y organizaciones en todo el planeta. Las estadísticas con respecto a las cifras de contagiados y fallecidos es alarmante. Esto ha obligado a muchos gobiernos de diversos países a cerrar fronteras, aeropuertos y aislar a la población para evitar que esta enfermedad se siga propagando. La COVID-19 ha afectado al sector asegurador en la comercialización de los productos, en lo digital, en lo laboral y en las acciones sociales y reputación (Montalbo et al., 2020).

De acuerdo con un informe emitido por Deloitte las empresas del sector privado han realizado donaciones para las personas y comunidades más necesitadas y han optado por brindar ayuda financiera al pago de las primas de sus clientes lo cual no afectará a los asegurados ni a su cobertura (Montalbo et al., 2020). La actividad digital (e-commerce) ha aumentado en las empresas de seguros, lo que ha generado nuevos lazos de comunicación y contratación con los clientes y entre colaboradores. Sin embargo, hubo una desaceleración en las primas (ingresos), cuyos efectos se prolongarán de dos a tres años (Montalbo et al., 2020). Así mismo, las empresas de seguros y planes de entidades prestadoras de salud (EPS) peruanas afirmaron que, durante el periodo de pandemia, todos los asegurados contarían con atención médica al 100\% (Asociación Peruana de Empresas de Seguros, 2019). Ello revela una decisión socialmente responsable de las empresas peruanas.

Los seguros son esenciales para las personas y empresas porque brindan garantía en su vida o actividad cotidiana, tanto para estos como para su familia y propiedades, ya que nos ofrecen tranquilidad, protección, reducción de riesgos cuando suceda algún tipo de tragedia o catástrofe e incluso promueve el ahorro (Superintendencia de Banca, Seguros y AFP, 2016). Debido a la importancia de los seguros, este estudio se realizó en la empresa Pacífico Seguros, domiciliada en el distrito de San Isidro, ubicada en el ranking de las cinco mejores aseguradoras del Perú con más de medio siglo de actividad. Su misión ha sido asistir a sus clientes salvaguardando su estabilidad financiera y su visión, ser la aseguradora más destacada en América Latina con colaboradores altamente aptos e inspirados.

Desde otra perspectiva, en el Perú existen empresas con el Distintivo Empresa Socialmente Responsable 2019 como Calidda, Arca Continental Lindley, Sodimac, Backus, Pacifico, Motored, Pacasmayo, Ferreyros CAT, BCP, entre otras. Es así que en el Perú es el único sello que brinda un valor agregado a la reputación y competitividad institucional relacionada con las mejoras prácticas en desarrollo sostenible y responsabilidad social y calidad (Perú 2021, 2019).

En concordancia con lo anterior, la calidad puede ser entendida como las discrepancias entre las percepciones del consumidor sobre un determinado bien o servicio ofrecido y las expectativas sobre dicho servicio (Loranca-Valle et al., 2019) y en ese marco de ideas la gestión de calidad, no solo debe apuntar al manejo idóneo y pulcro de la mercancía o servicio que se brinda, sino que los errores en los procedimientos sean $0 \%$ y darles la máxima importancia a las personas relacionándolas a la gestión de la responsabilidad social por medio del tiempo y la madurez (Deulofeu, 2006).

$\mathrm{Al}$ respecto, en el Perú, solo un 1\% del total de las empresas formales tienen un sistema de gestión de la calidad, una de ellas es el Organismo Superior de la Inversión de Energía y Minería, que recibió el premio Nacional de Calidad en el 2019 y una medalla Líder en la Categoría de Oro, debido a que han sistematizado sus procesos y medido sus resultados, lo que ha producido bienestar en la organización y mejorado la calidad de sus servicios energéticos (Cisneros, 2019). La no puesta en práctica de este tipo de 
sistemas en la mayoría de empresas en el país se debe a que no se lo ha integrado al círculo de la calidad como tampoco creado una nueva norma técnica peruana y brindado mayor importancia al Instituto Nacional de Calidad, factores que contribuirán a la mejora de la competencia en el mercado peruano que ayuden a mejorar la vida y sostenibilidad del entorno.

\subsection{Teorías de responsabilidad social}

Entre las diversas teorías sobre la responsabilidad social podemos citar a la de Bowen (1953) estudiado por Ormaza et al. (2020) cuya propuesta era disminuir a largo plazo los problemas económicos de la sociedad si los objetivos de una organización empresarial son alcanzados. Así mismo, la propuesta del mismo autor concuerda con el análisis de Duque et al. (2013) ya que los empresarios si bien tienen como objetivo lo económico su finalidad es social, aunque Ferrero (2014), al estudiar a Friedman (1970), concluye que los daños producidos en la sociedad son externalidades y su solución cae bajo la responsabilidad del gobierno que debe procurar la solución más eficaz; esta posición es interpretada por Duque et al. (2013), quienes, en relación con el mismo autor, argumentan que esos problemas los deben solucionar las fuerzas del mercado.

En relación con lo anterior, Carroll (1979), citado por Duque et al. (2013) establece que la Responsabilidad Social Empresarial (RSE) considera las percepciones jurídicas, ético-morales, económicas que la sociedad posee de las empresas en una época determinada. Esta posición se complementa con la de Licandro et al. (2019), al citar al mismo autor (Carrol, 1985) en el sentido de que la gestión socialmente responsable gestiona los impactos dentro de la organización o al exterior buscando proteger el entorno y producir mejoras en este.

Para efectos de esta investigación en relación con los conceptos de responsabilidad social citamos a Verduzco (2006), quien la considera como la predisposición de una organización para escuchar, entender y atender los requerimientos de los ciudadanos contribuyendo al desarrollo interno y su impacto positivo en el entorno. Asimismo, consideramos la responsabilidad en el medio ambiente como otro de los conceptos a considerar, que se convierte en el compromiso que tiene una organización con respecto al cuidado del entorno (Oxfam Intermón, 2016) y responsabilidad con la comunidad como la contribución que realiza la organización para darle solución a los impactos que esta genera y por las distintas problemáticas que existen como pobreza extrema, desigualdad social, corrupción, entre otras (Uribe et al., 2005).

\subsection{Teorías de gestión de la calidad}

En referencia a las diversas teorías de gestión de la calidad, Lizarzaburu (2016), citado por Amaya et al. (2020), indica que este sistema ha evolucionado en el tiempo pasando de enfoques de satisfacción plena de los consumidores y usuarios; de la inspección al control de calidad; el aseguramiento; hasta modernamente desarrollarse como gestión de calidad y calidad de los sistemas integrados de gestión. Por lo indicado, consideramos lo expresado por Hernández et al. (2018), citando a Goetsch y Davis (2014), que es un mecanismo trascendental para planificar, controlar y perfeccionar la calidad en las empresas, lo que concuerda con lo expuesto por Morán (2016), citando el ciclo de Deming y Traba et al. (2020), que a su vez cita el trabajo de Peter Drucker titulado "Vida de la nueva organización", quien sostiene que la empresa del mañana es como una orquesta sinfónica, donde prima la alta especialización individual con coordinación y sincronismo. Finalmente, Hernández et al. (2018), citando a Hernández (2011), señalan que la calidad debe relacionarse indubitablemente como integrante de las 
estrategias de responsabilidad social que genere mejoras sociales y del medio ambiente como la colocación de productos o servicios que sean idóneos para los clientes.

Para efectos de nuestra investigación, en relación con las definiciones de gestión de la calidad consideramos la de Camisón et al. (2006) como factores, directivas, normas, capacidades, conocimientos, metas, entre otras, por las cuales el empresario establece planes de acción, gestiona, ejecuta y controla diversas actividades para alcanzar los logros establecidos. Adicionalmente, consideramos como otro concepto en esta investigación el del nivel de desempeño laboral como la evaluación, el rendimiento y el actuar anterior y vigente de los trabajadores en relación con sus estándares (Dessler \& Varela, 2011); y el nivel de seguridad y salud de los empleados como el sistema que se encarga de reconocer, evaluar, cortar y eliminar los peligros que puede ocasionar accidentes, incidentes o enfermedades en los empleados, lo que puede ser manejado por parte del empleador, pero también por parte de los colaboradores, quienes deben recibir capacitación para poder colaborar con este (Tovalino, 2017).

\subsection{Dimensiones, principios y medición de las variables objeto de estudio}

Según Levante (2015), el tema de la responsabilidad social no es solo un instrumento de marketing sino un compromiso real con la sociedad, que fortalece la reputación de la organización. Esta ayuda a planificar la estrategia y gestionar conocimiento, que se integra a la forma de pensar de los integrantes de la organización. Los principios que identifican a las empresas que practican las políticas de responsabilidad social son: a) cumplir la legislación internacional y nacional, b) ética organizacional, c) satisfacer las necesidades de los grupos de interés, d) equilibrio social, económico, y medioambiental y e) brindar información. Estos lineamientos son considerados por diversas entidades como Pacto Mundial, la OCDE, la Organización Internacional del Trabajo y las normas sobre Responsabilidad Social de la Unión Europea contenidas en su Libro Verde (Puentes et al., 2020).

La responsabilidad social cuenta con dos dimensiones: intrínseca y extrínseca. La dimensión intrínseca se relaciona con los trabajadores, el medio ambiente, con la coordinación de materias primas, con las condiciones de trabajo o con el peligro que pueden correr cuando elaboran los productos o brindan los servicios. Mientras que la dimensión extrínseca se refiere a la sociedad, proveedores, consumidores, clientes, entre otros, que se vinculan al negocio y a los que se le debe crear un valor. La empresa puede pertenecer a cualquier de estas dimensiones, pero todas tienen necesidades comunes como (i) la transparencia informativa; (ii) la participación y (iii) el beneficio mutuo (Fernández, 2010).

Dentro de esta dimensión (extrínseca) se encuentra el medioambiente la cual se guía a través de la norma ISO 26000 que se rige por siete ejes verticales, la dirección del negocio, la jurisprudencia, la praxis laboral, el medio ambiente, las prácticas adecuadas de las tareas, materia de compradores y colaboración activa en la mejora de la comunidad (Estévez, 2015). Para medir el medio ambiente, se pueden usar los siguientes instrumentos: la huella de carbono o la huella hídrica. La primera se mide mediante un análisis de emisiones GEI durante un año u otro periodo determinado, la cual se realiza a través de la acumulación de un inventario de estudios (Gerendas, 2018).

Otro aspecto a considerar es la responsabilidad con la comunidad y para medir la satisfacción de las comunidades con los proyectos que realizan las empresas se emplean encuestas y entrevistas, dependiendo del tamaño de la comunidad ya que si es una comunidad grande se pueden realizar encuestas con escala de Likert. De ser una comunidad pequeña se puede realizar de manera personal a cada una de las familias por medio de entrevistas y un cuestionario más específico (Uribe et al., 2005). Esta acti- 
vidad bien puede catalogarse como publicidad social (Cárdenas-Rebelo \& Orosco-Toro, 2020), que tiene que ver con la percepción y aceptación que también las comunidades sobre el desarrollo de las empresas, el entendimiento de sus demandas y requerimientos, sus carencias y las perspectivas de estas, lo que se constituirá en información de interés para las empresas, pero es muy caro y para algunas empresas inaccesible sobre todo para las mypes (Romero, 2018). Un caso relacionado con este análisis es el realizado por Barrio (2016) en la empresa Unilever, en el que se demostró que mediante las campañas que realizaba la empresa se logró impactar a más de diez millones de personas por medio de la creación de puestos de trabajos, concientizar a las personas en sus hábitos de salud y de bienestar. Sin embargo, nos indica Reyes (2017) que en muchas empresas con políticas medioambientales estas no son utilizadas por los empleados, porque no hay un plan de capacitación y de acompañamiento que permita promover la cultura del medioambiente empresarial.

Toda empresa cuenta con tres aspectos en los que debe influir la responsabilidad social corporativa: el ambiental, el económico y el social (Puentes et al., 2020). Por el lado ambiental, la explotación de materias del planeta su depredación, la existencia de desechos industriales y domésticos, y los productos biodegradables que afectan al planeta han obligado a crear las denominadas finanzas ambientales, cuyo objetivo es reducir estas amenazas surgiendo los certificados de emisiones reducidas (CER) y el mercado de carbono que son difundidos por el Consejo Ejecutivo del Mecanismo de Desarrollo Limpio, que se monetizan a fin de año. Su propósito es la reducción de emisión suficiente de este compuesto contaminante al menor costo (Estévez, 2015). Si se logra encontrar un equilibrio entre estos, se puede mejorar el desarrollo sostenible y gestionar de una mejor manera los recursos naturales y los humanos, que es la base de la continuidad y el éxito de la empresa (Antelo \& Robaina, 2015). Cada empresa, al desarrollar productos o servicios, afecta el medio ambiente de manera particular y por ello su misión es establecer estrategias para evitar, reducir correctivos, medidas de control, así como capacitar adecuadamente al personal para contribuir con el entorno (Pérez et al., 2016).

En referencia al aspecto económico, este se refiere a la inversión para la adquisición de bienes o recursos que requieren para generar utilidades y convertirse, a nivel del mercado, en fuertes competidores que generen beneficios en el inmediato, mediano y largo plazo a aquellos con las que se relaciona. Mientras que, en el aspecto social, debe integrarse por buenas prácticas a los colaboradores como condiciones remunerativas y laborales adecuadas, capacitación correcta basada en valores que la empresa sostiene y practica como el apoyo a su comunidad (Puentes et al., 2020).

En el estudio de Tarí y García (2009) se destaca que existen ocho dimensiones que merecen ser identificadas: la primera el liderazgo, que involucra el ejercicio de poder a cargo del empresario por lo que los miembros obedecerán las órdenes aun cuando puedan asistir a la toma de decisiones. La segunda es la planificación, o la sucesión de pasos para alcanzar objetivos. La tercera es la gestión de empleados, que implica la formación de los trabajadores y sus equipos o herramientas a emplear. La cuarta es la gestión de proceso, que contempla la coordinación y administración del perfeccionamiento de los procedimientos. La quinta es la información y análisis, que implica el estudio de la cualidad, la medición y el análisis comparativo; la sexta es el enfoque al cliente, que estudia la estrategia que vincule con los usuarios. La séptima es la gestión de proveedores, que interpreta la relación que tienen los negocios con los abastecedores; y por último, el diseño del producto, que cuantifica la colaboración de todas las áreas afectadas con la estructura del producto y sus verificaciones. 
La ISO 9001:2015, en la sección 9.1.1, exige la medición de este sistema como requisito al declarar que toda empresa debe valorar el performance y su efectividad. Cada organización puede decidir lo que más se ajuste a sus necesidades; sin embargo, existen medidas para cuantificar el rendimiento del sistema como (a) la satisfacción de los clientes; (b) usar factores que midan acorde con su elaboración; (c) la rentabilidad y el incremento de las ganancias indican si los índices financieros son buenos o no; y (d) la tasa de rendimiento la cual, al medir este indicador las empresas contemplan que se mida la efectividad del sistema de gestión de calidad (IsoTools Excellence, 2017).

No obstante, para mantener el mejoramiento continuo de este sistema debe vigilarse mediante las auditorías. De igual forma, debe documentarse y evidenciar registros obligatorios, físicos o electrónicos, como son el reglamento interno de trabajo; el Sistema de Seguridad y Salud Ocupacional, los criterios para identificar accidentes e incidentes riesgosos, medidas de prevención, entre otros; registros de inspecciones, registros de exámenes médicos ocupacionales, entre otros (Tovalino, 2017).

\subsection{Analogía y relación entre la responsabilidad y la gestión de calidad}

Mientras que la calidad busca satisfacer la necesidad de los clientes, la responsabilidad social procesa y atiende las expectativas de los grupos de interés (Morán, 2016). Ambas variables no se improvisan, son el resultado de un largo camino que las empresas recorren por medio del análisis e implementación que realizan los gerentes y jefes de primera línea, los cuales a través del cambio constante en el mundo deben adaptarse a los nuevos desafíos y el avance tecnológico. Así mismo, el ánimo de comprometerse de los colaboradores de una entidad con las comunidades menos privilegiadas se relaciona con el estudio de Henao (2014), que demostró la necesidad de contar con un área de responsabilidad social en la entidad para con ello lograr una rentabilidad financiera exitosa. La responsabilidad social y la calidad van de la mano, ya que para ambas se puede aplicar la propuesta de Deming. El autor indica que para ambos es imperativo responder de manera ágil y eficaz a las $5 \mathrm{~W}$ y $1 \mathrm{H}$, por sus siglas en inglés: ¿qué?, ¿por qué?, ¿dónde?, ¿cuándo?, ¿quién?, y ¿cómo? Esto solo es de manera teórica, pero es indispensable que los socios y gerentes de estas empresas contraten personal capacitado que cuenten con altos estándares de ética, en lo personal y profesional; y, con valores notables, puesto que esto produce un lazo más fuerte entre la gestión de calidad y la responsabilidad social según lo afirman Morán (2016) y Navarro (2015) permitiendo una selección y desarrollo de herramientas, que con un enfoque de proceso, integra aspectos organizativos y tecnológicos (Pacheco et al., 2021).

Finalmente, las dos variables bajo estudio se vinculan en la plena ejecución de la denominada gestión del conocimiento, ya que las organizaciones que manejan adecuadamente la información y le dan valor tienen una ventaja al aplicar sus políticas impactando de manera positiva en la misma empresa como fuera de ella. Así, según Echeverry et al. (2018), la gestión del conocimiento coadyuva en la forma de gestionar e incentivar el desarrollo de ideas innovadoras y útiles ya que fomenta la creatividad esencial para el cambio y la competencia.

En el mismo sentido lo manifiestan Agudelo y Valencia (2018), al indicar que el conocimiento incorporado en la empresa le da valor ayudando a formar ventajas competitivas que deben traducirse en lo productivo. Ello permite coadyuvar en la ejecución de los dos temas objeto de estudio por existir plena relación entre las denominadas estrategias económicas que buscan colocar productos o servicios en el mercado con las simbólicas que tienen como finalidad acercar a la organización al entorno para reducir la percepción negativa que puede tener el impacto empresarial producto de su desarrollo e influencia (Pellegrin et al., 2018). 


\section{Materiales y método}

El objetivo principal de este estudio fue determinar la relación entre la responsabilidad social y la gestión de calidad en una empresa peruana de seguros, mientras que los objetivos específicos fueron: determinar la relación entre la responsabilidad en la comunidad y la gestión de calidad, y la responsabilidad y el medio ambiente con la gestión de calidad en una empresa peruana de seguros.

Es una investigación descriptiva correlacional, de paradigma positivista de enfoque cuantitativo, de alcance transversal (Rivera-Camino, 2014) y diseño no experimental con el propósito de realizar una investigación básica (Hernández et al., 2010). La población y muestra de la investigación de esta empresa peruana de seguros está conformada por 2387 colaboradores, de los cuales el $68 \%$ es de género femenino y el 32\% de género masculino; $48,8 \%$ pertenecen al área de gerentes, subgerentes y personal administrativo; $43,6 \%$ al área de fuerza de ventas; y, el 7,6\% son practicantes. Los colaboradores trabajan en Lima. Mientras que para hallar la muestra se aplicó la fórmula del muestreo probabilístico aleatorio simple, la cual tuvo un resultado de 331 colaboradores.

Se utilizó una encuesta de 25 preguntas con escala de Likert de cinco niveles que, según Llaurado (2014), se convierte en una herramienta para medir actitudes y el grado de conformidad de los encuestados ante las afirmaciones que se les plantea. En la tabla 1, se describe la operacionalización de las variables, dimensiones e indicadores del presente estudio.

Se aplicó la validación de contenidos por juicio de expertos, conformado por cuatro docentes investigadores de la Universidad de Piura con grados de maestros y doctores en la Escuela de Administración de Empresas. Así como dos especialistas de la empresa investigada, el subgerente de Responsabilidad Social y la consultora de relaciones públicas de la empresa. Para realizar la validación, se consideran tres indicadores: claridad, conveniencia y alcance. Según Cochachi (2018), una investigación es fiable si el coeficiente alfa es mayor a 0,7 . Cuando se procesó el cuestionario por el sistema SPSS nos dio un resultado de 0,962 , lo cual indicaba que el instrumento presenta una alta confiabilidad a través del Cronbach alfa.

\section{Resultados}

Al realizar un análisis descriptivo se contempla la conducta de ambas variables para posteriormente analizar la explicación de la información recogida. Se encuestaron un total de 331 colaboradores que fueron seleccionados de manera aleatoria, utilizando el muestreo aleatorio simple.

\section{Tabla 1. Responsabilidad social por dimensiones}

\begin{tabular}{|l|c|c|c|}
\hline & $\begin{array}{c}\text { Responsabilidad } \\
\text { social }\end{array}$ & $\begin{array}{c}\text { Dimensión } \\
\text { responsabilidad } \\
\text { con la comunidad }\end{array}$ & $\begin{array}{c}\text { Dimensión } \\
\text { responsabilidad } \\
\text { en el medio } \\
\text { ambiente }\end{array}$ \\
\hline N & 331 & 331 & 331 \\
\hline Media & 38,12 & 18,12 & 19,99 \\
\hline Desviación & 8,250 & 2,879 & 5,763 \\
\hline Mediana & 43,00 & 19,00 & 23,00 \\
\hline
\end{tabular}

Fuente: Elaboración propia. 
En la Tabla 1, se aprecia que la responsabilidad social obtuvo un puntaje promedio de $38,12 \pm 8,25$ y un puntaje mediano de 43 , lo que indica que el $50 \%$ de colaboradores presentan un puntaje por encima de 43; el puntaje máximo obtenido es de 50 puntos. Por lo tanto, los colaboradores de la empresa de seguros presentan una alta responsabilidad social frente a la comunidad y el cuidado del medio ambiente.

Tabla 2. Gestión de la calidad por dimensiones

\begin{tabular}{|l|c|c|c|}
\cline { 2 - 4 } \multicolumn{1}{c|}{} & $\begin{array}{c}\text { Gestión de la } \\
\text { calidad }\end{array}$ & $\begin{array}{c}\text { Dimensión nivel } \\
\text { de seguridad con } \\
\text { los colaboradores }\end{array}$ & $\begin{array}{c}\text { Dimensión nivel de } \\
\text { desempeño de los } \\
\text { colaboradores }\end{array}$ \\
\hline $\mathrm{N}$ & 331 & 331 & 331 \\
\hline Media & 34,49 & 16,74 & 17,75 \\
\hline Desviación & 7,282 & 4,338 & 3,306 \\
\hline Mediana & 38,00 & 18,00 & 19,00 \\
\hline
\end{tabular}

Fuente: Elaboración propia.

En la Tabla 2, se aprecia que la gestión de la calidad obtuvo un puntaje promedio de $34,49 \pm 7,28$ y un puntaje mediano de 38 , lo que indica que el $50 \%$ de colaboradores presentan un puntaje por encima de este valor, siendo el puntaje máximo obtenido de 45 puntos. Por lo tanto, los colaboradores de la empresa de seguros presentan un alto nivel de seguridad y desempeño.

Tabla 3. Rho de Spearman para responsabilidad social con la gestión de calidad

\begin{tabular}{|c|c|c|c|c|}
\hline & & & $\begin{array}{c}\text { Responsabilidad } \\
\text { social }\end{array}$ & $\begin{array}{l}\text { Gestión de } \\
\text { la calidad }\end{array}$ \\
\hline \multirow{6}{*}{$\begin{array}{l}\text { Rho de } \\
\text { Spearman }\end{array}$} & \multirow{3}{*}{$\begin{array}{l}\text { Responsabilidad } \\
\text { social }\end{array}$} & $\begin{array}{l}\text { Coeficiente de } \\
\text { correlación }\end{array}$ & 1,000 &, $721 * *$ \\
\hline & & Sig. (bilateral) & . & , 000 \\
\hline & & $\mathrm{N}$ & 331 & 331 \\
\hline & \multirow{3}{*}{$\begin{array}{l}\text { Gestión de } \\
\text { la calidad }\end{array}$} & $\begin{array}{l}\text { Coeficiente de } \\
\text { correlación }\end{array}$ &, $721 * *$ & 1,000 \\
\hline & & Sig. (bilateral) & , 000 & . \\
\hline & & $\mathrm{N}$ & 331 & 331 \\
\hline
\end{tabular}

** La correlación es significativa en el nivel 0,01 (bilateral).

Fuente: Elaboración propia

Se evaluó la relación entre la gestión de la calidad y la RSE a partir del cálculo del coeficiente correlación de Spearman, obteniéndose un coeficiente de 0,721, lo que indica una relación alta entre las dos variables y positiva. Es decir, a mayor RSE, mayor gestión de la calidad en la empresa de seguros. 


\section{Tabla 4. Rho de Spearman para responsabilidad con la comunidad y las dimensiones de la gestión de calidad}

\begin{tabular}{|l|l|l|l|c|}
\cline { 3 - 6 } & & & & \\
\hline
\end{tabular}

** La correlación es significativa en el nivel 0,01 (bilateral).

Fuente: Elaboración propia

En la Tabla 4, se evaluó la relación entre la dimensión responsabilidad de la comunidad con las dimensiones de la gestión de la calidad, al evaluar la relación de responsabilidad con la comunidad y la seguridad con los colaboradores se obtuvo un coeficiente de correlación de Spearman de 0,756, lo que indica una alta relación entre ambas dimensiones, siendo de relación positiva. Es decir, a mayor responsabilidad con la comunidad mayor nivel de seguridad de los colaboradores. Así también observamos una fuerte relación entre el nivel de seguridad con el nivel de desempeño de los colaboradores $($ rho $=0,601 ; p=0,000)$, siendo esta relación positiva, es decir a mayor nivel de seguridad mayor nivel desempeño. 


\section{Tabla 5. Rho de Spearman para Responsabilidad en el medio ambiente y las dimensiones de la gestión de calidad}

\begin{tabular}{|c|c|c|c|c|c|}
\hline & & & \\
\hline & & & 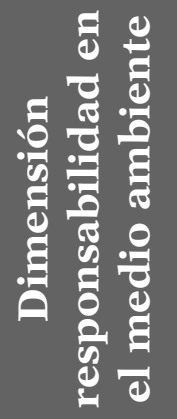 & 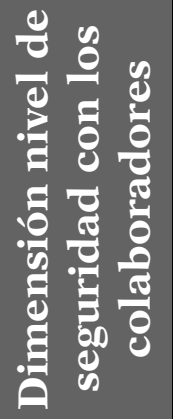 & 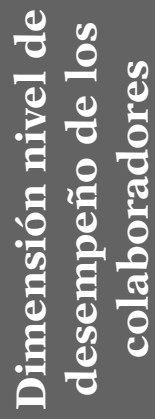 \\
\hline \multirow{9}{*}{$\begin{array}{l}\text { Rho de } \\
\text { Spearman }\end{array}$} & \multirow{3}{*}{$\begin{array}{l}\text { Dimensión respon- } \\
\text { sabilidad en el me- } \\
\text { dio ambiente }\end{array}$} & Coeficiente de correlación & 1,000 &, $571 * *$ &, $782 * *$ \\
\hline & & Sig. (bilateral) & . & 000 &, 000 \\
\hline & & $\mathrm{N}$ & 331 & 331 & 331 \\
\hline & \multirow{3}{*}{$\begin{array}{l}\text { Dimensión nivel de } \\
\text { seguridad con los } \\
\text { colaboradores }\end{array}$} & Coeficiente de correlación &, $571 * *$ & 1,000 & $601 * *$ \\
\hline & & Sig. (bilateral) & ,000 & . & ,000 \\
\hline & & $\mathrm{N}$ & 331 & 331 & 331 \\
\hline & \multirow{3}{*}{$\begin{array}{l}\text { Dimensión nivel de } \\
\text { desempeño de los } \\
\text { colaboradores }\end{array}$} & Coeficiente de correlación &, $782 * *$ & ,601** & 1,000 \\
\hline & & Sig. (bilateral) & 000 &, 000 & - \\
\hline & & $\mathrm{N}$ & 331 & 331 & 331 \\
\hline
\end{tabular}

** La correlación es significativa en el nivel 0,01 (bilateral).

Fuente: Elaboración propia

En la Tabla 5, se evaluó la relación entre la dimensión responsabilidad del medio ambiente con las dimensiones de la gestión de la calidad. Al evaluar la relación de responsabilidad en el medio ambiente y la seguridad con los colaboradores se obtuvo un coeficiente de correlación de Spearman de 0,571, lo que indica una alta relación entre ambas dimensiones, siendo de relación positiva. Es decir, a mayor responsabilidad en el medio ambiente mayor nivel de seguridad de los colaboradores.

Los modelos de gestión de calidad han ido incorporando elementos de la responsabilidad social para atender las necesidades de los grupos de interés, no obstante, toman elementos de integridad, equidad, transparencia, cuidado y libertad; son elementos también de la responsabilidad, cada vez se entrelazan más y más (Tari \& García, 2009).

\section{Discusión y conclusiones}

\subsection{Discusión}

En la tabla 1, se aprecia que, en relación con la responsabilidad social el 50\% de colaboradores de la empresa de seguros presentan una alta responsabilidad social frente a la comunidad y el cuidado del medio ambiente. Asimismo, en la tabla 2, se aprecia que en referencia a la gestión de la calidad el 50\% de colaboradores presentan un puntaje alto lo que implica que los colaboradores de la empresa de seguros presentan un alto nivel de seguridad y desempeño. Estos resultados tienen relación con la política desarrollada por los gerentes al promover las campañas de responsabilidad social en la empresa. A la vez concuerdan con los hallazgos de las investigaciones realizadas (Levante, 2015; Henao, 2014), comprobando también la existencia del ciclo de Deming citado por Morán (2016) 
como un sistema integrado expuesto por Hernández et al. (2018) citando a Goetsch y Davis (2014) y se relaciona con lo propuesto por Hernández et al. (2018) citando a Hernández (2011) que permita generar con la RSE valor a la empresa.

En relación con los resultados de la tabla 3, al evaluarse la relación entre la gestión de la calidad y la RSE se revela que existe una relación alta entre las dos variables y positiva, es decir, a mayor RSE mayor gestión de la calidad en la empresa de seguros. Este resultado se evidencia debido a la motivación que la empresa propicia a los colaboradores con talleres de capacitación sobre salud y trabajo con el objetivo preventivo de evitar algún tipo de accidente laboral (Dessler \& Varela, 2011) y (Tovalino, 2017), en el sentido de mejoras en las condiciones laborales; lo que conduce según Navarro (2015) y Pacheco et al. (2021) a establecer un sistema orgánico entre las variables analizadas en esta investigación para lograr los procedimientos o técnicas adecuadas y alcanzar una sostenibilidad en el tiempo. El resultado se evidencia también con la posición de Bowen (1953) sostenida por Ormaza et al. (2020) en el sentido que el mercado puede solucionar problemas del entorno. Asimismo, la mayoría de los colaboradores indicó que son voluntarios de los programas de responsabilidad social porque están convencidos que su ayuda mejorará a la comunidad (Antelo \& Robaina, 2015) sus propias vidas, la de sus familias y la comunidad, concordando con lo expuesto por Carroll (1979), citado por Duque et al. (2013) en el sentido que la RSE impacta al interior o exterior de la empresa por los estudios desarrollados (Uribe et al., 2005; Verduzco, 2006).

De acuerdo con los resultados de la tabla 4, se evaluó la relación entre la dimensión responsabilidad de la comunidad con las dimensiones de la gestión de la calidad, de lo que resultó una relación positiva. Es decir, a mayor responsabilidad con la comunidad, mayor nivel de seguridad de los colaboradores y existe una fuerte relación entre el nivel de seguridad con el nivel de desempeño de los colaboradores; es decir, a mayor nivel de seguridad, mayor nivel de desempeño. Al respecto, se puede apreciar que sí existe una asociación con la gestión de calidad estudiada por Barrio (2016) al analizar el impacto de las campañas de la empresa Unilever en la creación de puestos de trabajos y en la concientización en sus hábitos de salud y de bienestar que se relaciona al análisis del cuidado del medio ambiente de Oxfam Intermón (2016); y la posición de Traba et al. (2020) al citar a Drucker cuando expresa que las empresas deben comportarse con calidad para integrar colaboradores y entorno, lo que revela la seguridad y el desempeño laboral relacionado con lo expresado por Tovalino (2017) y Dessler y Varela (2011), en el sentido de que se debe potenciar las estrategias simbólicas (Pellegrin et al., 2018), con lo que se manejará y gestionará adecuadamente el conocimiento (Echeverry et al., 2018) de la organización. Esto da valores agregados que permite innovar.

En función de los resultados de la tabla 5, se evidenció una alta relación entre la dimensión responsabilidad del medio ambiente con las dimensiones de la gestión de la calidad la seguridad; es decir, a mayor responsabilidad en el medio ambiente mayor nivel de seguridad de los colaboradores. Esto se relaciona con la posición teórica establecida por Pérez et al. (2016), quienes indicaron que para que exista la estabilidad empresarial con comportamiento responsable sus integrantes, deben actuar conscientemente respetando las normas y el entorno incluido el medio ambiente. Esta dependencia que existe entre la responsabilidad en la comunidad y la gestión de calidad se demostró en los análisis obtenidos por Reyes (2017), en donde se evidencia lo contrario, ya que según el indicado autor muchas organizaciones poseen políticas medioambientales, pero no se concretan debido a la falta de motivación, capacitación o simplemente desconocimiento lo que puede ocurrir en la empresa bajo análisis. 


\subsection{Conclusiones}

Por lo indicado, se evidencia que sí existe relación entre la responsabilidad social y la gestión de calidad, porque a lo largo de estos años la empresa ha establecido y ejecutado políticas relacionadas con las variables estudiadas, esto ha logrado convencer progresivamente a muchos colaboradores de la necesidad de que participen en lo que se le cataloga como una entidad con calidad de sostenible alineada con la línea principal del negocio.

El nivel de satisfacción en los colaboradores es importante porque la empresa incide en su permanente capacitación con cursos de seguridad, salud en el trabajo para evitar accidentes laborales, entre otros programas de capacitación, que les permita alcanzar metas laborales, pero sobre todo por el hecho de estar integrando una entidad prestigiosa y sostenible.

Asimismo, se evidencia relación entre la responsabilidad social y el medio ambiente porque está muy ligada a la línea principal del negocio por medio de la compra de los bonos de carbono, que provienen de la reserva de Tambopata, que ha logrado disminuir la emisión de $\mathrm{CO} 2$ producto de la energía que consume la empresa como el uso de las computadoras, el aire acondicionado, entre otros. A ello se suman las campañas de reciclaje y el apoyo a las campañas para la preservación del medio ambiente a pesar de que la mayoría de colaboradores no participa.

Existe relación entre la gestión de la calidad y la responsabilidad social en la comunidad, porque ha tenido reconocimiento en las comunidades de humilde condición en distritos como Villa María del Triunfo o San Juan de Lurigancho en Lima, que influyen en la salud de estas familias como el caso de las denominadas ponchilas para niños que eran mochilas con un poncho para ayudar a combatir el friaje cuando los niños de zonas remotas de los andes iban a las escuelas y la instrucción a las familias sobre salud, accidentes personales y la preservación del entorno ambiental.

La principal limitación que posee la organización es que aún le falta trabajar en el desarrollo de estrategias simbólicas, es decir gestionar el conocimiento de temas que motiven a los colaboradores e involucren también a los proveedores a participar convencidos de los beneficios que poseen los programas de responsabilidad y gestión de calidad, para con ello acercar más a la empresa al entorno y reducir la percepción negativa que genera impacto organizacional sobre todo en esta etapa de pandemia y en la que se generará posteriormente a la era de la COVID-19, en la que las organizaciones se enfrentarán a niveles de competencia mayores. Por lo indicado, sugerimos investigar el desarrollo de estrategias que desarrollan diversas entidades para revertir este tipo de limitaciones.

\section{Referencias}

Agudelo, E., \& Valencia, A. (2018). La gestión del conocimiento, una política organizacional para la empresa de hoy. Ingeniare. Revista chilena de ingeniería, 26(4), 673-684. https://bit.ly/2Z5QAI6

Amaya, P., Félix, E., Rojas, S., \& Díaz, L. (2020). Gestión de la calidad: un estudio desde sus principios. Revista Venezolana de Gerencia, 25(90), 632-647. https://doi.org/10.37960/rvg.v25i90

Antelo, Y., \& Robaina, D. (2015, enero-abril). Análisis de la responsabilidad social empresarial basado en un modelo de lógica difusa compensatoria. Ingeniería Industrial, 36(1), 58-69. https://bit.ly/2Z7b2Zj

Asociación Peruana de Empresas de Seguros (2019). Apeseg. Asociación de Empresas de Seguros. https://www.apeseg.org.pe/

Barrio, E. (2016). La gestión de responsabilidad corporativa: Caso Unilever España [tesis doctoral, Universidad Autónoma de Barcelona]. Tesis Doctorals en Xarxa. https://bit.ly/3jDavI9.

Camisón, C., Cruz, S., \& González, T. (2006). Gestión de la calidad: conceptos, enfoques, modelos y sistemas. 
Pearson Educación. https://bit.ly/3jFrObj

Cárdenas-Rebelo, A., \& Orosco-Toro, J. (2020, octubre-marzo). Publicidad social y su influencia en las campañas sociales de prevención de accidentes de tránsito. Retos, 10(20), 219-231. https://doi.org/10.17163/ret.n20.2020.02

Cisneros, S. (2019). Osinergmin obtuvo premio nacional a la calidad. Horizonte Minero. https://bit.ly/3ab0IpF

Cochachi, V. (2018). La responsabilidad social y la gestión empresarial de la empresa Saga Falabella, año 2017 [Tesis de maestría, Universidad César Vallejo]. Repositorio Digital Institucional. https://bit.ly/3jGFwea

Deulofeu, J. (2006). Gestión de calidad total en el comercio detallista. Ediciones Pirámide.

Dessler, G., \& Varela, R. (2011). Administración de recursos humanos (L.E. Pineda Ayala; V. Campos Olguín; 2. ${ }^{\mathrm{a}}$ ed.). Pearson Educación. https://bit.ly/2Z69Sxl

Duque, Y., Cardona, M. \& Rendón, J. (2013, julio-diciembre) Responsabilidad social empresarial: teorías, índices, estándares y certificaciones. Cuadernos de Administración, 29(50), 196-206. https://bit.ly/372qJ8k

Echeverry, A, Lozada, N., \& Arias, J. (2018). Incidencia de las prácticas de gestión del conocimiento sobre la creatividad organizacional. Información Tecnológica, 29(1), 71-82. https://bit.ly/3tNFPs5.

Estévez, R. (2015, 24 de julio). ¿En qué consiste la norma ISO 26000? Eco Inteligencia. https://bit.ly/3d3W7Y3

Fernández, R. (2010, 6 de julio). Dimensiones de la responsabilidad social empresarial. Sus actores. Papel de las políticas públicas. Diario Responsable. https://bit.ly/3rKseA5

Ferrero, I. (2014, octubre-diciembre). Responsabilidad social y responsabilidad limitada en Milton Friedman. De un modelo shareholder hacia un modelo stakeholder. Revista de Ciencias Sociales, 20(4), 633-643. https://dx.doi.org/10.2139/ssrn.2629838

Gerendas, S. (2018, 11 de diciembre). Huella de carbono: ¿Qué es y cómo se mide? Gestiopolis. https://bit.ly/2Ze1lrT

Henao, J. (2014). La responsabilidad social empresarial como estrategia de gestión en la organización Pranha S. A. [tesis de maestría, Universidad Nacional de Colombia]. Repositorio institucional. Biblioteca Digital. https://bit.ly/3b1LpyO

Hernández, H., Barrios, I., \& Martínez, D. (2018, enero-junio). Gestión de la calidad: elemento clave para el desarrollo de las organizaciones. Criterio Libre, 16(28), 179-195. https://bit.ly/3qe7qjZ

Hernández, R., Fernández, C., \& Baptista, P. (2010). Metodología de la Investigación (5. ${ }^{a}$ ed.). McGraw Hill.

IsoTools Excellence (2017, 12 de diciembre). Consejos prácticos para medir el sistema de gestión de calidad según ISO 9001. ISO Tools Excellence. https://bit.ly/2Ng0Rz5.

Llaurado, O. (2014). La escala de Likert: qué es y cómo utilizarla. Netquest. https://bit.ly/2MOlimU

Levante (2015). Responsabilidad social, más que una estrategia de marketing. Levante: el mercado valenciano. https://bit.ly/2KYKkhV

Licandro, O., Alvarado-Peña, L., Sensores, E., \& Navarrete, J. (2019). Responsabilidad social empresaria: hacia la conformación de una tipología de definiciones. Revista Venezolana de Gerencia, 24(85), 281-299. https://doi.org/10.37960/revista.v24i85

Loranca-Valle, C, Cuesta-Valiño, P., \& Núñez-Barriopedro, E. (2019). Gestión de calidad como estrategia clave de la felicidad en el deporte federado. Retos, 9(18), 203-218. https://doi.org/10.17163/ret.n18.2019.02.

Montalbo, J., Ortiz, I., Herrero, J.L., \& Trejo, M. (2020). Impacto de la COVID-19 en el sector asegurador. Deloitte. https://bit.ly/2Zap506

Morán, R. (2016). Calidad y RSE: analogía y complementariedad. Quality Magazine, 189, 8-9. https://bit.ly/2LHzXzD

Navarro, X. (2015, 15 de septiembre). ¿Qué es la gestión de calidad? Deusto Formación. https://bit.ly/3d3Xwhh

Ormaza, J., Ochoa, J., Ramírez, F., \& Quevedo, J. (2020). Responsabilidad social empresarial en el 
Ecuador: abordaje desde la Agenda 2030. Revista de Ciencias Sociales 26(3), 175-193. https://bit.ly/3qbW7cf

Oxfam Intermón (2016). Responsabilidad social ambiental: la conciencia colectiva para mejorar el mundo. Oxfam Intermón. https://bit.ly/3pcwYgn

Pacheco, E., Gómez, B., Rodríguez, G., Cardoso, N., \& Araldi, J. (2021). Mejora de la calidad de la gestión de la energía: un análisis de las industrias en un país en desarrollo. Revista de la Uni-

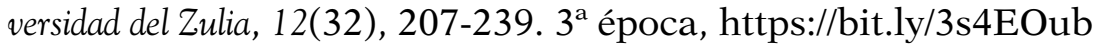

Pellegrin, G., Fleker, L., Pasquale, M., \& Ferrero, A. (2018, junio). Conflictos territoriales y estrategias empresariales en materia de responsabilidad social empresarial (RSE): repensando categorías de análisis. Cuaderno Urbano, 24(24), 73-91. http://dx.doi.org/10.30972/crn.24242922.

Pérez, M.J., Espinoza, C., \& Peralta, B. (2016). La responsabilidad social empresarial y su enfoque ambiental: Una visión sostenible a futuro. Revista Universidad y Sociedad, 8(3), 169-178. https://bit.ly/3rKy04J

Perú 2021 (2019). Distintivo Empresa Socialmente Responsable. Perú 2021. https://bit.ly/372401m

Puentes, R., Antequera, J.M., \& Velasco, M. d. (2020). La responsabilidad social corporativa y su importancia en el espacio europeo de educación superior. En J. Pindado-García (Ed.), Estableciendo puentes en una economía global. Universidad Jaén.

Reyes, L. (2017). Responsabilidad ambiental en la empresa del sector público: Caso dirección de impuestos y aduanas nacionales Santa Marta en perspectiva de los saberes del manejo de residuos sólidos [tesis de maestría, Universidad de Manizales]. Repositorio Institucional. https://bit.ly/3740qik

Rivera-Camino, J. (2014). Cómo escribir y publicar una tesis doctoral. ESIC.

Romero, M. (2018, 26 de octubre). ¿Responsabilidad social o compromiso con la comunidad? El Mundo. https://bit.ly/3rJkvlL

Superintendencia de Banca, Seguros y AFP (2016). Para vivir seguros: "Educación financiera para ti". Superintendencia de Banca, Seguros y AFP. https://bit.ly/3d0XYg7

Tarí, J., \& García, M. (2009) Dimensiones de la gestión del conocimiento y de la gestión de la calidad: una revisión de la literatura. Investigaciones Europeas de Dirección y Economía de la Empresa, 15(3), 139-152. https://doi.org/10.1016/S1135-2523(12)60105-1.

Tovalino, F. (2017). Obligaciones del empleador sobre salud y seguridad en el trabajo. Gaceta Jurídica.

Traba, L., Barletta, M., \& Velázquez, J. (2020). Teoría (y práctica) de las organizaciones. Ediciones Universidad Nacional del Litoral. https://bit.ly/3jHtGRa

Uribe, A., Gagelman, C., Pacheco, I., Gálvez, L., \& Peláez, A. (2005). Ética, responsabilidad social y empresa. Centro Editorial Universidad del Rosario.

Verduzco, A. (2006), Responsabilidad social empresarial: de la dimensión corporativa a la personal. The Anáhuac Journal, 6(1), 100-111. 